\title{
Special Issue Editorial: Advances on Scalable Information Systems for Big Data (InfoScale 2014)
}

\author{
Jason J. Jung ${ }^{1}$
}

Published online: 7 May 2015

(C) Springer Science+Business Media New York 2015

\section{Editorial}

We are in era of "Big Data". As data and knowledge volume keep increasing while global means for information dissemination continue to diversify, new methods, modeling paradigms and structures are needed to efficiently mount scalability requirements [1]. In the last few years, we have seen the proliferation of the use of heterogeneous distributed systems, ranging from simple Networks of Workstations, to highly complex grid computing environments. Such computational paradigms have been preferred due to their reduced costs and inherent scalability, which pose many challenges to scalable systems and applications in terms of information access, storage and retrieval. Cluster computing [2], cloud computing technology [3], data and knowledge bases, distributed information retrieval technology [4] and networking technology [5] should all converge to address the scalability concern. Furthermore, with the advent of emerging computing architectures (e.g., SMTs, GPUs, and multicores), the importance of designing techniques explicitly targeting these systems is becoming more and more important.

The 5th International Conference on Scalable Information Systems (InfoScale) has mainly focused on a wide array of scalability issues and investigated new approaches to tackle problems arising from the ever-growing size and complexity of information of all kinds.

This special issue features six selected papers with high quality from InfoScale held in Seoul, Korea, September 25-

Jason J. Jung

j2jung@gmail.com

1 Knowledge Engineering Laboratory, Department of Computer Engineering, Chung-Ang University, 84 Heukseok-ro, Dongjak-gu, Seoul, Republic of Korea
26, 2014. The first paper, entitled "Real-time Event Detection on Social Data Stream", presents an interesting framework to process big social data. Particularly, by analyzing the data streams from social media, useful events can be detected and applied to provide users with intelligent context-aware services.

The second paper "Similarity Searching for the Big Data" authored by Pavel Zezula considers the scalable framework for big data processing. The paper firstly finds out a necessity to discover descriptive information of complex and heterogeneous objects to make them accessible. Second, multimodal search structures are requested to efficiently conduct complex similarity queries possibly in outsourced environments while preserving privacy.

The third paper "Weighted Similarity Schemes for High Scalability in User-Based Collaborative Filtering" by Pirasteh et al. presents a novel recommender system (RecSys) based on similarity integration method. Different from conventional RecSys (which is based on single similarity between a pair of users), the proposed system collects all possible similarities between two users, and integrates them for representing the relationship between them in the best manners.

The fourth paper "A Novel Ranking Model for a Large-Scale Scientific Publication" investigates how to rank academic publications (e.g., research papers, conference proceedings, and journals), which are modeled in very highly complex networks. Given a large amount of publication data, authors have proposed multi-layered network model (call N-star model) to process.

The sixth paper "Multi-modal Similarity Retrieval with Distributed Key-value Store" by David Novak studies distributed system architecture by using key-value store. Particularly, with designing several search aspects (called, modalities), the study shows an efficient information retrieval performance for a large amount data (e.g., CoPhIR benchmark dataset).

The last paper "Big Bibliographic Data Analytics by Random Walk Model" by Jason J. Jung shows an interesting probabilistic 
approach to analyze a large amount of bibliographic data for providing users with various publication (and Citation) services. Particularly, the proposed method is based on random work for measuring citation networks among the research papers.

The guest editors are thankful to our reviewers for their effort in reviewing the manuscripts. We also thank the Editin-Chief, Dr. Imrich Chlamtac for his supportive guidance during the entire process.

\section{References}

1. Chen M, Mao S, Liu Y (2014) Big data: a survey. Mob Netw Appl 19(2):171-209

2. Jiang H, Chen Y, Qiao Z (2015) Scaling up mapreduce-based big data processing on multi-GPU systems. Clust Comput 18(1):369-383

3. Hashem IAT, Yaqoob I, Anuar NB (2015) The rise of "big data" on cloud computing: review and open research issues. Inf Syst 47:98-115

4. Long NH, Jung JJ (2015) Privacy-aware matching online social identities for multiple social networking services. Cybern Syst 46(1-2):69-83

5. Pham XH, Jung JJ (2014) Recommendation system based on multilingual entity matching on linked open data. J Intell Fuzzy Syst 27(2):589-599

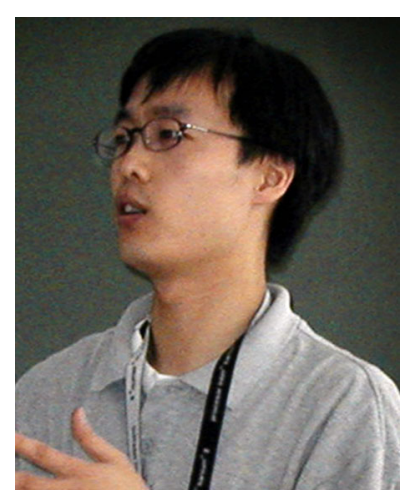

Dr. Jason J. Jung is an Associate Professor in Chung-Ang University, Korea, since September 2014. Before joining CAU, he was an Assistant Professor in Yeungnam University, Korea since 2007. Also, He was a postdoctoral researcher in INRIA Rhone-Alpes, France in 2006, and a visiting scientist in Fraunhofer Institute (FIRST) in Berlin, Germany in 2004. $\mathrm{He}$ received the B.Eng. in Computer Science and Mechanical Engineering from Inha University in 1999. He received M.S. and Ph.D. degrees in Computer and Information Engineering from Inha University in 2002 and 2005, respectively. Dr. Jung serves as Editorial board member of many international journals, e.g., Journal of Universal Computer Science, International Journal of Intelligent Information and Database Systems, International Journal of Social Network Mining and International Journal of Web Engineering and Technology. He has edited 10 special issues in international journals, 2 conference proceedings. He is the author of about 100 international publications. His research topics are knowledge engineering on social networks by using many types of AI methodologies, e.g., data mining, machine learning, and logical reasoning. Recently, he have been working on intelligent schemes to understand various social dynamics in large scale social media (e.g., Twitter and Flickr). 